\title{
Spin-Hall magnetoresistance and spin Seebeck effect in spin-spiral and paramagnetic phases of multiferroic $\mathrm{CoCr}_{2} \mathrm{O}_{4}$ films
}

\author{
A. Aqeel, ${ }^{1}$ N. Vlietstra, ${ }^{1}$ J. A. Heuver, ${ }^{1}$ G. E. W. Bauer, ${ }^{2,3}$ B. Noheda, ${ }^{1}$ B. J. van Wees, ${ }^{1}$ and T. T. M. Palstra ${ }^{1, *}$ \\ ${ }^{1}$ Zernike Institute for Advanced Materials, University of Groningen, Nijenborgh 4, 9747 AG Groningen, The Netherlands \\ ${ }^{2}$ Institute for Materials Research and WPI-AIMR, Tohoku University, Sendai, Miyagi 980-8577, Japan \\ ${ }^{3}$ Kavli Institute of NanoScience, Delft University of Technology, Lorentzweg 1, 2628 CJ Delft, The Netherlands
}

(Received 18 June 2015; revised manuscript received 21 September 2015; published 8 December 2015)

\begin{abstract}
We report on the spin-Hall magnetoresistance (SMR) and spin Seebeck effect (SSE) in multiferroic $\mathrm{CoCr}_{2} \mathrm{O}_{4}$ (CCO) spinel thin films with Pt contacts. We observe a large enhancement of both signals below the spinspiral $\left(T_{s}=28 \mathrm{~K}\right)$ and the spin lock-in $\left(T_{\text {lock-in }}=14 \mathrm{~K}\right)$ transitions. The SMR and SSE responses in the spin lock-in phase are one order of magnitude larger than those observed at the ferrimagnetic transition temperature $\left(T_{c}=94 \mathrm{~K}\right)$, which indicates that the interaction between spins at the Pt|CCO interface is more efficient in the noncollinear magnetic state. At $T>T_{c}$, magnetic-field-induced SMR and SSE signals are observed, which can be explained by a high interface susceptibility. Our results show that the spin transport at the Pt|CCO interface is sensitive to the magnetic phases but cannot be explained solely by the bulk magnetization.
\end{abstract}

DOI: 10.1103/PhysRevB.92.224410 PACS number(s): 72.15.Gd, 72.20.Pa, 72.25.Mk, 72.25.Pn

\section{INTRODUCTION}

Ferromagnetic (ferrimagnetic) insulators (FMIs) with metallic contacts that support the spin-Hall effect (SHE) and its inverse (ISHE) open new functionalities in the field of spintronics. The SHE refers to a charge current that induces a transverse spin current, which can be injected to actuate a metallic or insulating ferromagnet. The ISHE converts a spin current pumped out of a ferromagnet into a transverse charge current in the normal metal (NM). These concepts have been confirmed by many experiments on FMI|NM bilayers of a magnetic insulator (usually yttrium iron garnet (YIG)) and a heavy NM (usually platinum), for example, spin-pumping by ferromagnetic resonance [1-4], the spin Seebeck effect (SSE) [5-8], the spin Peltier effect [9], and the spin-Hall magnetoresistance (SMR) [10-15]. In the SMR, both the SHE and the ISHE act in a concerted manner to allow electrical detection of the FMI magnetization direction. The SSE refers to the conversion of thermal excitations of the magnetic order parameter (spin waves or magnons) into a spin current pumped into a NM and detected by the ISHE.

The SSE and SMR have been investigated up to now only for a limited number of insulating ferrimagnets (garnets and spinels) with collinear magnetizations and, recently, in an antiferromagnetic insulator [16]. However, magnetic insulators come in a large variety of magnetic orders. Especially fascinating are noncollinear magnets with competing magnetic interactions (spin frustration) induced by competing nextnearest-neighbor exchange. Alternatively, the spin-orbit coupling, such as the Moriya-Dzyaloshinskii interaction, favors complex spiral configurations and skyrmion order. While the coupling of noncollinear magnetizations with spin, charge, and heat transport is currently one of the hottest subjects in magnetism, its role in the SMR and SSE appears not to have been studied yet.

\footnotetext{
*t.t.m.palstra@rug.nl
}

Noncollinear magnetism emerges when the second-nearestneighbor magnetic interactions are of the same order as the first one, generating geometrical frustration that favors spin canting. Various spin-spiral orders, like proper screw, cycloidal, longitudinal-conical, and transverse-conical spiral, have been observed. The cycloidal and transverse-conical spiral orders break the inversion symmetry and induce a spontaneous electrical polarization, making these spiral magnetic systems multiferroic [17-21]. Here, we focus on noncollinear and multiferroic $\mathrm{CoCr}_{2} \mathrm{O}_{4}(\mathrm{CCO})$ thin films, reporting, to the best of our knowledge, the first experimental observation of the SMR and SSE in the $\mathrm{Pt} \mid \mathrm{CCO}$ system for a wide range of temperatures including the ferrimagnetic and spin-spiral phases.

$\mathrm{CCO}$ is one of the rare multiferroic materials with linear magnetoelectric coupling [22,23]. It has a normal spinel structure with three sublattices. $\mathrm{Co}^{2+}$ ions are located exclusively at the tetrahedral sites (forming one sublattice), while $\mathrm{Cr}^{3+}$ ions reside at the octahedral sites (in two sublattices). $\mathrm{Cr}^{3+}$ ions form a pyrochlore lattice with magnetic geometrical frustration [24,25]. Bulk CCO exhibits long-range collinear ferrimagnetic order below $T_{c}=93-95 \mathrm{~K}$ [24,26,27], with an easy axis of magnetization along the [001] direction, as illustrated in Figs. 1(a) and 1(c). At $T_{s}=28 \mathrm{~K}$, the ferrimagnetic long-range order adopts an additional short-range spiral order as illustrated in Figs. 1(b) and 1(c), which is known as the spinspiral phase transition. The conical spiral has $48^{\circ}, 71^{\circ}$, and $28^{\circ}$ cone angles with the [001] direction for the $\mathrm{Co}, \mathrm{Cr}$ I, and $\mathrm{Cr}$ II sublattices, respectively. Below $T_{s}$, magnetic spin spirals move oxygen atoms off-center due to the inverse DzyaloshinskiiMoriya interaction [26,28], which results in the appearance of spontaneous electrical polarization. At $T_{\text {lock-in }}=14 \mathrm{~K}$, the spin spiral becomes commensurate with the lattice by spin-lattice coupling, which is known as the spin lock-in transition.

Here, we report a systematic study of the SMR and SSE in Pt|CCO bilayers at low to room temperature $(T=5-300 \mathrm{~K})$. At each temperature, we record the dependence on the angle of an in-plane applied magnetic field. We observe strong effects of the CCO magnetic order, with the largest signals in the spin lock-in phase at $T<14 \mathrm{~K}$. 


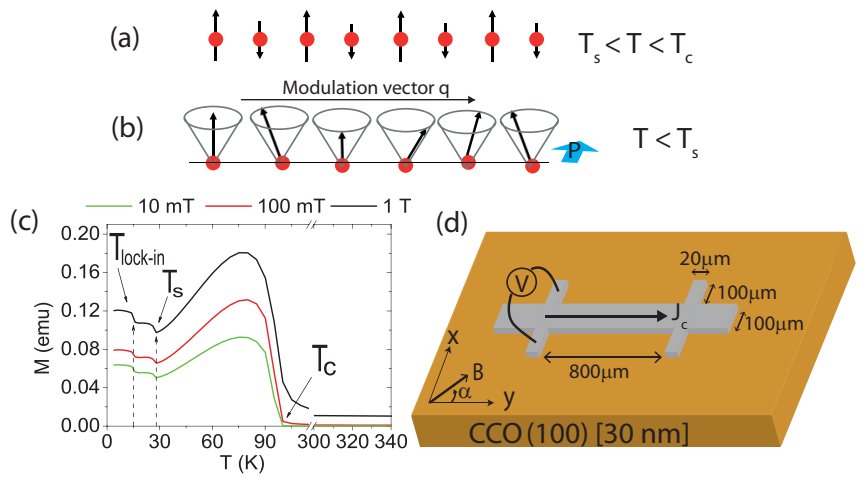

FIG. 1. (Color online) (a, b) Impression of the types of magnetic order in CCO. (a) Ferrimagnetic state for $T_{s}<T<T_{c}$ and (b) transverse conical spiral state for $T<T_{s}$ with an electrical polarization P. Here, $T_{c}$ and $T_{s}$ are the Curie transition temperature to a collinear ferrimagnetic phase and the spin-spiral transition temperature, respectively. (c) Temperature dependence of the zerofield cooled magnetization of the CCO target powder used for film deposition for different applied magnetic fields. $T_{\text {lock-in }}$ is the spin lock-in magnetic transition temperature. (d) Device configuration for the transverse resistance (planar Hall effect) measurement of the $\mathrm{Pt}$ film on top of CCO.

\section{SAMPLE GROWTH AND CHARACTERISTICS}

CCO films (30 nm thick) were grown on (001) $\mathrm{MgO}$ substrates by pulsed laser deposition. Sintered ceramic CCO targets [29] were ablated with a KrF excimer laser light with a wavelength of $248 \mathrm{~nm}$ and a repetition frequency of $0.5 \mathrm{~Hz}$. During deposition, the $\mathrm{MgO}$ substrates are kept at $500{ }^{\circ} \mathrm{C}$ in an oxygen plasma atmosphere, having a base pressure of 0.01 mbar. Afterwards, the films were cooled down, at $5{ }^{\circ} \mathrm{C}$ per minute in a 0.5 bar $\mathrm{O}_{2}$ atmosphere. Before further device fabrication, the films were annealed at $200{ }^{\circ} \mathrm{C}$ for $60 \mathrm{~min}$ in an $\mathrm{O}_{2}$ atmosphere. The crystal structure of the $\mathrm{CCO}$ films was determined by $\mathrm{x}$-ray diffraction, where the rocking curves show a high crystalline quality with a full width at half-maximum (FWHM) below $0.03^{\circ}$.

The magnetization of the CCO films was measured with a SQUID magnetometer. The films show an in-plane magnetic anisotropy with a coercive field $H_{c}$ around $2 \mathrm{~T}$. For temperatures below $50 \mathrm{~K}$, the magnetic transitions are hard to detect due to the large paramagnetic substrate background compared to the small magnetic moment of the thin film (see Appendix A). Nevertheless, SQUID measurements [30,31] and $\mathrm{x}$-ray resonance magnetic scattering both indicate the same magnetic transitions in thin films as reported for bulk CCO. In order to demonstrate the magnetic transitions in $\mathrm{CCO}$, we measured the temperature dependence of the magnetization of bulk CCO targets used for film deposition. Figure 1(c) shows a magnetization of the CCO targets with the same transitions as reported in the literature [32]. We carried out transport experiments on Hall-bar structures patterned by electron beam lithography onto which a $4 \mathrm{~nm}$ thick Pt film was deposited by dc sputtering, as shown in Fig. 1(d).

\section{MEASUREMENT TECHNIQUES}

The SMR and SSE have been measured simultaneously by using the lock-in detection technique [33]. Using two Stanford
SR-830 lock-in amplifiers, the first and second harmonic voltage responses were recorded separately. To minimize the background voltage, we used the transverse instead of the longitudinal configuration, as shown schematically in Fig. 1(d), which is also referred to as the "planar Hall" voltage. The SMR signals scale linearly with the applied current and therefore are detected in the Pt resistance of the first harmonic response [33]. The current-induced SSE scales quadratically with the applied current and therefore is detected in the second harmonic response. The angular dependence of the SMR and SSE were studied by rotating an external magnetic field in the plane of the film [34], which, above $T_{c}$, induces magnetization or (if large enough) aligns the direction of the CCO magnetization below $T_{c}$. The in-plane angle $\alpha$ of the magnetic field is defined relative to the current direction along the $y$-axis as indicated in Fig. 1(d). All experiments were carried out in a quantum design PPMS system, at magnetic fields $B \geqslant 2 \mathrm{~T}$ and for temperatures from 5 to $300 \mathrm{~K}$.

\section{RESULTS AND DISCUSSION}

\section{A. Spin-Hall magnetoresistance}

Owing to the SHE, an ac current through the Pt Hall bar creates an accumulation of ac spin at the Pt|CCO interface, which can be partially absorbed or fully reflected, depending on the interface magnetization $\vec{M}$ of the FMI [see Figs. 2(a) and 2(b)]. The reflected spin currents generate an extra charge current via the ISHE, thereby reducing the resistance. While the longitudinal resistance establishes the maximum modulation between these two configurations with $\alpha=0^{\circ}$ or $90^{\circ}$, the planar Hall effect vanishes. In contrast, when $\alpha=45^{\circ}$, the ISHE is maximal [35], as sketched in Fig. 2(c). The additional electromotive force (emf) scales with the applied current and is therefore, detected by the first harmonic transverse resistance $R_{1}$ [33], defined as $V_{1} / I$, where $V_{1}$ is the first harmonic signal of the lock-in amplifier generated by the applied ac current of amplitude $I$ (see Appendix B for more details).

The $\alpha$ dependence of the first harmonic response of $R_{1}$ at $5 \mathrm{~K}$ in Fig. 2(d) shows, in addition to the expected SMR, an ordinary Hall effect generated by a magnetic-field component normal to the film due to a slight misalignment of the sample, by an angle $\beta$. The ordinary Hall effect voltage has a $\sin (\alpha+\phi)$ angular dependence, where the phase $\phi$ is governed by the sample tilt direction. A prefactor of $0.7 \mu \mathrm{V}$ at a current of $2 \mathrm{~mA}$ in a magnetic field of $6 \mathrm{~T}$ corresponds to a tilt of $\beta<2^{\circ}$. The ordinary Hall voltage of $\mathrm{Pt} \mid \mathrm{CCO}$ is nearly temperature independent and scales linearly with the applied current and magnetic field, as expected. After subtracting the ordinary Hall effect from $R_{1}$, the anticipated $\sin 2 \alpha$ dependence associated with the SMR remains [10], as illustrated in Fig. 2(d). The SMR signal, $R_{1(2 \alpha)}^{\mathrm{ampl}}$, is defined as the amplitude of the $\sin 2 \alpha$ component and plotted in Figs. 2(e)-2(g) as a function of the temperature in magnetic fields of 6, 4, and $2 \mathrm{~T}$, respectively. Exchanging the current and voltage probes in the Hall bar in a field of $6 \mathrm{~T}$ leads to identical SMR profiles, confirming that the interface magnetization is parallel to the applied field. The SMR signals of CCO and yttrium iron garnet (YIG) are compared in Appendix C. 

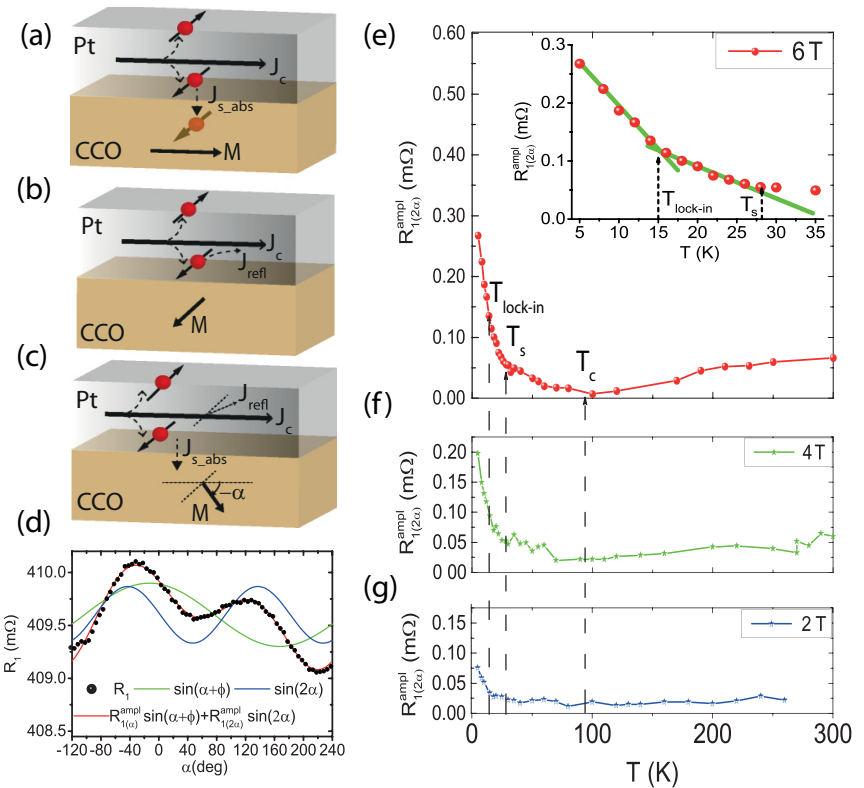

FIG. 2. (Color online) Illustration of the SMR in Pt|CCO bilayers. The charge current $I$ induces a spin current and thereby spin accumulation at the Pt $\mid \mathrm{CCO}$ interface by virtue of the SHE. (a) This spin accumulation is absorbed as a spin transfer torque when the magnetization $\vec{M}$ is perpendicular to the current-induced spin polarization in Pt. (b) When $\vec{M}$ is parallel to the spin accumulation, the spin current is reflected back into Pt, where it generates an additional charge current, $J_{\text {refl }}$, by the ISHE. (c) When $\vec{M}$ is at an angle $\alpha$ to $J_{c}$, the component of the spin accumulation perpendicular to $\vec{M}$ is absorbed and the component parallel is reflected, leading to an extra charge current component normal to $J_{c}$ that is detected in the Hall configuration. (d) Angular dependence of the first harmonic response in the transverse configuration, $R_{1}=V_{1} / I$, for $I=2 \mathrm{~mA}$ at $5 \mathrm{~K}$ in an applied magnetic field of $6 \mathrm{~T}$. The $\sin (\alpha+\phi)$ and $\sin 2 \alpha$ curves illustrate the additive contributions from the ordinary Hall effect and the SMR. (e-g) Temperature dependence of the SMR $R_{1(2 \alpha)}^{\mathrm{ampl}}$ for $J_{c}=2 \mathrm{~mA}$ at $6 \mathrm{~T}(\mathrm{e}), 4 \mathrm{~T}(\mathrm{f})$, and $2 \mathrm{~T}(\mathrm{~g})$. Here, $R_{1(2 \alpha)}^{\mathrm{ampl}}$ is the amplitude of the $\sin 2 \alpha$ component from the SMR. $T_{c}, T_{s}$, and $T_{\text {lock-in }}$ are the ferrimagnetic, spin-spiral, and spin lock-in magnetic transition temperatures, respectively. Inset in (e): Zoomed-in image of the SMR signal below $T_{s}$ and $T_{\text {lock-in }}$ at $6 \mathrm{~T}$.

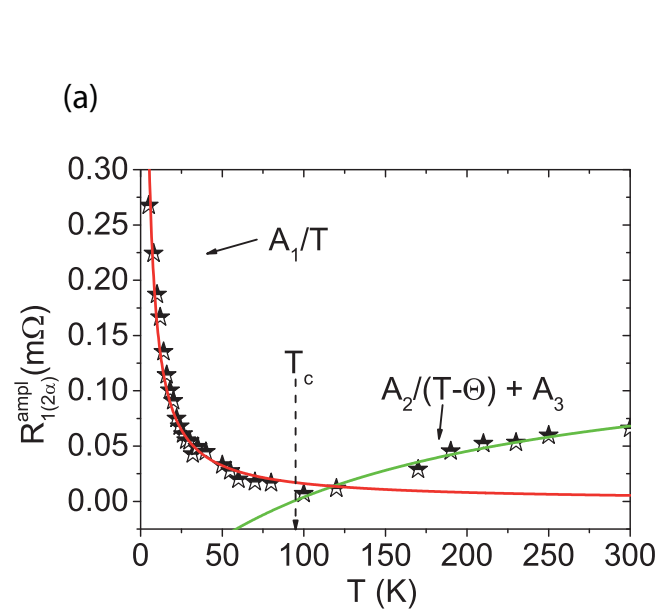

Below the collinear ferrimagnetic transition temperature, $T_{c}=94 \mathrm{~K}$, the SMR signal increases with decreasing temperature. The inset in Fig. 2(e) recorded at a magnetic field of $6 \mathrm{~T}$ shows a distinct change of slope at the spin-spiral transition temperature to a noncollinear magnetic phase, $T_{s}=28 \mathrm{~K}$. At $T<T_{s}$, the SMR signal is more than one order of magnitude larger than the signal observed at $T_{c}$. A further decrease in temperature below the spin lock-in transition temperature, $T_{\text {lock-in }}=14 \mathrm{~K}$, doubles the SMR compared to $T_{s}$. This observation indicates that the exchange interaction between metal and magnet in the $\mathrm{Pt} \mid \mathrm{CCO}$ system is more efficient in the noncollinear spiral phase than in the collinear ferrimagnetic phase. The maximum SMR signal is observed in the spin lock-in phase, when the period of the spin spiral becomes commensurate with the lattice. Below $T_{c}, R_{1}=A_{1} T^{-1}$ [see Fig. 3(a)] gives an excellent fit, where $A_{1}$ scales linearly with the applied magnetic field, as shown in Fig. 3(b). $A_{1}$ should be proportional to the interface spin-mixing conductance, which describes the ability of the ferromagnet to absorb or emit transverse polarized spin currents. When the interface magnetization vanishes, therefore, so does $A_{1}$. Above $T_{c}$, all magnetization is generated by the applied magnetic field and $A_{1}$ is a measure of the interface paramagnetic susceptibility. For a magnetic interface, we anticipate a bilinear $A_{1}(B)$, with a large slope at low magnetic fields that reflects the expulsion of magnetic domain walls. Extrapolation of the high-field data should lead to a finite cutoff at zero magnetic fields (as in studies of the anomalous Hall effect). However, the extrapolation of $A_{1}(B)$ does not lead to a statistically significant $A_{1}(0)$. At present we therefore cannot confirm whether the observed SMR signals reflect the paramagnetic susceptibility of a nonmagnetic interface or a spontaneous interface magnetization texture.

In order to shed more light on the $T$ dependence for $T<T_{c}$, we compare the SMR with the bulk magnetization, as shown in Fig. 1(c). The $T_{c}, T_{s}$, and $T_{\text {lock-in }}$ transition temperatures established from the SMR closely correspond to the transition temperatures observed for the bulk magnetization. In the spinspiral and spin lock-in phases, the bulk magnetization does not depend much on the temperature, as shown in Fig. 1(c), in

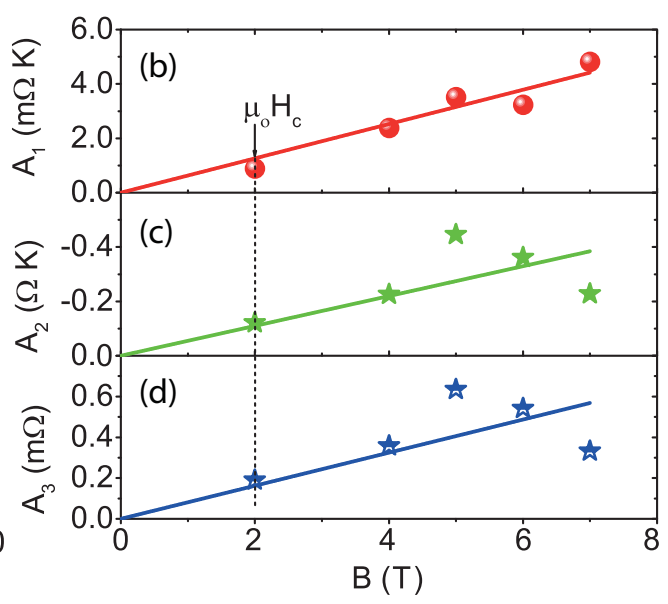

FIG. 3. (Color online) (a) Temperature dependence of $R_{1(2 \alpha)}^{\mathrm{ampl}}$ in a magnetic field of $6 \mathrm{~T}$ for $I=2 \mathrm{~mA}$. The leftward (red) curve shows the fit to the Curie law at low temperatures, below $\mathrm{T}_{c}$, and the rightward (green) curve shows the fit to the Curie-Weiss law in the high-temperature range, above $\mathrm{T}_{c}$ (for $\theta_{\mathrm{CW}}=-550 \mathrm{~K}$ ). (b-d) Field dependence of fitting parameters $A_{1}(\mathrm{~b}), A_{2}(\mathrm{c})$, and $A_{3}(\mathrm{~d})$. 
stark contrast to the SMR below $T_{s}$ [Fig. 2(e)]. According to the present understanding, the SMR does not directly reflect the bulk magnetization. Theoretically, it is generated by the modulation of the spin current at the NM|FMI interface by the magnetization direction [35] and is roughly proportional to the density of parallel- and/or antiparallel-oriented magnetic moments at the interface [36]. The SMR enhancement below $T_{s}$ thus reflects an increased (anti)ferromagnetic order of the magnetic moments at the interface. The induced interface magnetization can be generated by either Co ions, contributing to the ferrimagnetic component, or $\mathrm{Cr}$ ions, responsible for the cycloidal component of the spin spiral. The ferrimagnetic component saturates around $28 \mathrm{~K}$ and, therefore, cannot explain the enhanced SMR below $28 \mathrm{~K}$. However, neutron scattering experiments show that the spiral component below $T_{s}=28 \mathrm{~K}$ strongly depends on the temperature [24], similarly to the SMR signal. We therefore venture that the development of the spiral order should be explained by a strongly temperaturedependent ordering of $\mathrm{Cr}$ ions that does not contribute to the global magnetization. This implies that, by a simple transport experiment, we can distinguish ferrimagnetic from cycloidal components of the interface magnetization.

We observe a finite SMR in the paramagnetic phase for $T>T_{c}$, complementing reports on spin pumping [37] and the SSE [8] in the paramagnetic state. In CCO the magnetic susceptibility, for $T>T_{c}$, follows a Curie-Weiss law with a negative Curie-Weiss temperature, $\theta_{\mathrm{CW}}=-550 \mathrm{~K}$, which is evidence of antiferromagnetic correlations. The high ratio $\left|\theta_{\mathrm{CW}}\right| / T_{c} \approx 6$ indicates significant magnetic frustration due to competing sublattice exchange interactions in CCO [32], resulting in short-range order above $T_{c}$. The SMR signal above $T_{c}$ increases with the temperature until it saturates to a constant value around room temperature, as shown in Figs. 2(e)-2(g). The SMR signal for $T>T_{c}$ provides evidence of an unusual interface magnetic susceptibility of our films. We fit the SMR signals with the Curie-Weiss law, but a single fitting parameter, $A_{2}$, does not capture the contributions from the molecular fields. Very much unlike the bulk magnetic susceptibility, the SMR signal is suppressed at $T_{c}$, which can be taken care of by introducing an additional parameter, $A_{3}$, as shown in Fig. 3(a). Both fitting parameters, $A_{2}$ and $A_{3}$, scale linearly with the applied magnetic field, as shown in Figs. 3(c) and 3(d). This result supports our conclusion, drawn earlier, that the SMR signal cannot be explained solely by the bulk magnetization of $\mathrm{CCO}$, even in the paramagnetic phase. The temperatureindependent background modeled by $A_{3}$ and the negative sign of $A_{2}$ remain unexplained, which may indicate that the shortrange order reported for bulk CCO above $T_{c}$ is importantly modified at the interface with Pt.

\section{B. Spin Seebeck effect}

We now discuss the SSE signal observed in the second harmonic response using the lock-in detection technique as described in Ref. [33]. The SSE is caused by the Joule heating of the Pt Hall bar, generating a heat current into the ferromagnet which is absorbed by magnons. This heat current is associated with a spin current polarized along the magnetization direction, which can be detected electrically by the ISHE, as sketched in Fig. 4(a). The second harmonic response, $R_{2}=\sqrt{2} V_{2} / I^{2}$, where $V_{2}$ is the second harmonic signal of the lock-in amplifier at a phase set at $\phi=-90^{\circ}$ [33]. The observed second harmonic response of the transverse resistance obeys the $\sin \alpha$ angular dependence anticipated for the SSE, as shown in Fig. 4(b). We define the amplitude of the $R_{2}^{\mathrm{ampl}}=R_{2} / \cos \alpha$ for each temperature and magnetic-field strength. The SSE signal for $\mathrm{Pt} \mid \mathrm{CCO}$ has the same sign as that for Pt|YIG (see Appendix C). Figures 4(c)-4(e) show the temperature dependence of $R_{2}^{\mathrm{ampl}}$ in magnetic fields of 6,4 , and $2 \mathrm{~T}$, respectively.

In the collinear ferrimagnetic state at $T<T_{c}$, the SSE response increases with decreasing temperature. Figure 4(c) gives evidence of a large SSE enhancement below the spinspiral transition temperature $T_{S}$ and, again, below $T_{\text {lock-in. The }}$ SSE signal below $T_{s}$ is five times larger than the signal observed at $T_{c}$. The inset in Fig. 4(c) illustrates that the SSE at $T<T_{\text {lock-in }}$ increases by an order of magnitude from $T=80 \mathrm{~K}$. Moreover, below $T_{s}=28 \mathrm{~K}$, the SSE signal scales linearly with the applied magnetic field. The SSE is too noisy to provide as clear evidence for phase transitions at $T_{c}, T_{s}$, and $T_{\text {lock-in }}$ as the SMR signals do.

Conventional thermoelectric effects become small with decreasing temperatures, so what causes the observed remarkable enhancement at low temperatures? We can understand the temperature dependence of the SSE signal by considering the contributions from different magnetic sublattices [38,39]. CCO is a collinear ferrimagnet above $T_{s}$, with three sublattices associated with Co and oppositely polarized $\mathrm{Cr}$ I and $\mathrm{Cr}$ II ion moments [24], but without any magnetization compensation below $T_{c}$. The magnetic sublattices contribute to the SSE with correlated thermal fluctuations. The coupled sublattices have acoustic (ferromagnetic) and optical (antiferromagnetic) modes. The fundamental ferromagnetic modes govern the low-energy excitations that are probed by ferromagnetic resonance and Brillouin light scattering. The optical modes are shifted to a higher energy by the exchange interaction. In the collinear ferrimagnetic state close to $T_{c}$, the magnetic order and exchange interaction is still weak. The ferromagnetic mode then only slightly dominates the optical modes that are still substantially excited. The acoustic and optical modes contribute to the SSE with different signs and, close to $T_{c}$, cancel to a large extent. With decreasing temperature, the exchange splitting of the optical modes increases, and therefore, they become increasingly depleted. The suppression of thermal pumping of the optical modes therefore leads to an apparent enhancement of the SSE at lower temperatures. This mechanism explains [40] the low-temperature sign change of the SSE of the ferrimagnetic insulator $\mathrm{Gd}_{3} \mathrm{Fe}_{5} \mathrm{O}_{5}$ (GdIG) [39] as well as the apparent suppression of the SSE in YIG at temperatures above $300 \mathrm{~K}$ [41].

In contrast to collinear magnetic order, the magnetization texture of a spin spiral is sensitively modulated by an external magnetic field [42], which might be reflected by the observed magnetic-field dependence of the SSE below $T_{s}$, as shown in Figs. 4(c)-4(e). The increase in the SSE with applied magnetic field below $T_{s}$ should persist until the $\mathrm{Co}^{2+}$ and $\mathrm{Cr}^{3+}$ momenta of $3 \mu_{B}$ /ion are fully aligned. No signs of magnetization saturation were observed at magnetic fields up to $30 \mathrm{~T}$ [32], so it would be interesting to find out whether the SSE also can be enhanced by applying high magnetic fields. 


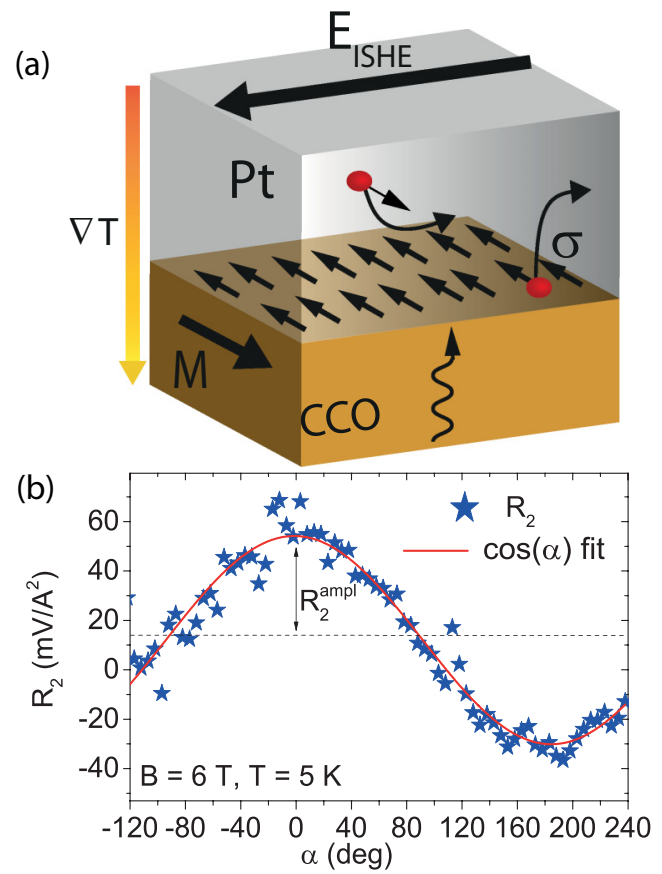

(c)

(d)
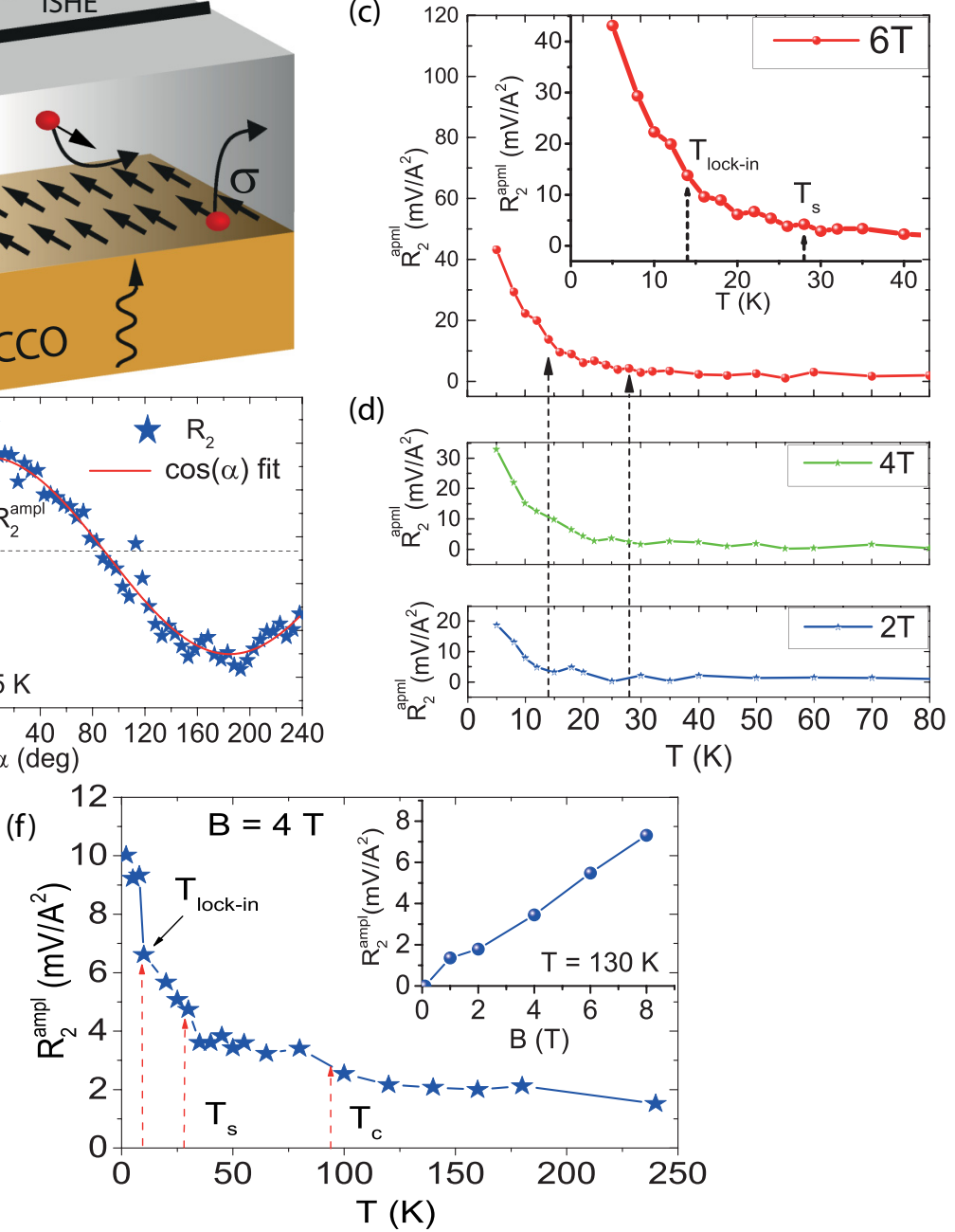

FIG. 4. (Color online) (a) Spin Seebeck effect in Pt|CCO. A thermal gradient creates magnons at the interface by absorption of a spin current from Pt with polarization $\vec{\sigma} \| \vec{M}$, where $\vec{M}$ is the CCO interface magnetization. The spin current generates an electromotive force $\vec{E}_{\mathrm{ISHE}}$ by the ISHE. (b) Angular dependence of the second harmonic response, $R_{2}=\sqrt{2} V_{2} / I^{2}$, at $5 \mathrm{~K}$ for $I=2 \mathrm{~mA}$ in an applied magnetic field of $6 \mathrm{~T}$. (c-e) Temperature dependence of $R_{2}^{\mathrm{ampl}}$ at $6 \mathrm{~T}$ (c), $4 \mathrm{~T}$ (d), and $2 \mathrm{~T}$ (e) for $I=2 \mathrm{~mA}$. Inset in (c): Plot emphasizing the enhancement of the SSE signal below the spin-spiral $\left(T_{s}\right)$ and spin lock-in $\left(T_{\text {lock-in }}\right)$ transition temperatures in a magnetic field of $6 \mathrm{~T}$. (f) Temperature dependence of $R_{2}^{\text {ampl }}$ in the nonlinear current regime $(I=5 \mathrm{~mA})$. Inset: Magnetic-field dependence of $R_{2}^{\text {ampl }}$ at $T=130 \mathrm{~K}$.

The observed correlation between the SMR and the SSE suggests that the spin-mixing conductance; i.e., the transport measure of the exchange interaction between ferromagnet and NM, plays an important role. Other factors, such asmagnetization damping and magnon transport to the interface, which is affected by the magnon-phonon interaction [43], may contribute to the observed enhancement of the SSE below $T_{s}$. A quantitative description of the SSE can be approached by atomistic spin simulations that take the full spin-wave spectrum associated with the three sublattices with different cone angles, chiralities, and damping parameters, as well as the spin-mixing conductances of the interface to $\mathrm{Pt}$, into account [40].

Above $T_{c}$ the SSE cannot be established at low heating current levels ( $I=2 \mathrm{~mA})$, as shown in Fig. 4(c). However, at a higher current $(I=5 \mathrm{~mA})$ in a magnetic field of $4 \mathrm{~T}$, a finite SSE signal is detected above $T_{c}$ and up to room temperature [see Fig. 4(f)]. At these current levels, $T_{\text {lock-in }}$ is shifted to a lower temperature by $4 \mathrm{~K}$ due to sample heating, and nonlinearities kick in; i.e., the SSE voltage $\nsim I^{2}$. The SSE still scales linearly with the applied magnetic field as shown for $T=130 \mathrm{~K}$ in the inset in Fig. 4(f). The presence of the SSE above $T_{c}$ can be explained by the large longitudinal magnetic susceptibility of CCO films, which is responsible for the SMR response as well [37].

In summary, by lock-in detection we simultaneously measured the SMR and SSE in Pt|CCO bilayers. The temperature dependence of the SMR and, though less so, the SSE, exposes distinct anomalies at the magnetic phase transitions. A remarkable enhancement of both SMR and SSE signals is observed at low temperatures $\left(T<T_{S}\right)$. SMR is more than one order of magnitude larger at $T<T_{\text {lock-in }}$ compared to the signals around $T_{c}$. We relate the observed enhancement of the SMR below $T_{s}$ to contributions from the cycloidal spiral projected onto the spin accumulation at the $\mathrm{Pt} \mid \mathrm{CCO}$ interface. The SSE signal also increases by a factor of 2 
when the temperature is lowered below $T_{s}$. The temperature dependence of the SSE does not simply reflect the bulk magnetization; instead the intricate magnetization dynamics of coupled sublattices needs to be considered. Our results suggest that the magnons associated with the Cr-sublattice magnetization texture plays an essential role in the SSE. Vice versa, we establish that the SMR and SSE are powerful instruments that complement ferromagnetic resonance and neutron scattering techniques to analyze the magnetization dynamics of complex oxides including multiferroics.

\section{ACKNOWLEDGMENTS}

We would like to acknowledge J. Baas, H. Bonder, M. de Roosz, and J. G. Holstein for technical assistance. This work was supported by the Foundation for Fundamental Research on Matter (FOM), NanoNextNL, a micro- and nanotechnology consortium of the government of The Netherlands and 130 partners, NanoLab NL, InSpin (EU-FP7-ICT Grant No. 612759), the Zernike Institute for Advanced Materials, Grantsin-Aid for Scientific Research (Nos. 25247056, 25220910, and 26103006), and DFG Priority Programme 1538 (BA 2954/2).

\section{APPENDIX A: CCO FILM MAGNETIZATION}

The temperature-dependent magnetization of the CCO film in Fig. 5 as measured by SQUID magnetometry is evidence of a phase transition at $T_{c}=94 \mathrm{~K}$ to collinear ferrimagnet order, but the spin-spiral and spin lock-in transitions are not visible, because of the small magnetization of a thin film as explained in the text. The apparent increase in magnetization below $20 \mathrm{~K}$ is probably caused by paramagnetic impurities in the substrate.

\section{APPENDIX B: LOCK-IN DETECTION}

All measurements reported in thetext are carried out using a lock-in detection technique [33] with $I \leqslant 5 \mathrm{~mA}$ ac current bias in the Pt film. The generated voltage can be expanded as

$$
V(t)=R_{1} I(t)+R_{2} I^{2}(t)+R_{3} I^{3}(t)+\ldots
$$

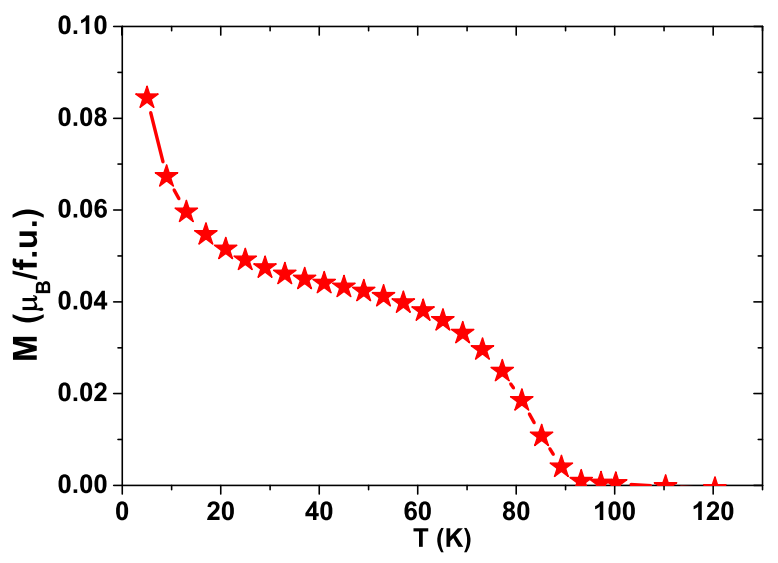

FIG. 5. (Color online) In-plane magnetization of a CCO film on a $\mathrm{MgO}$ substrate in a magnetic field of $0.01 \mathrm{~T}$ after cooling at $1 \mathrm{~T}$. where $R_{n}$ represent the $n$th harmonic response. For $I(t)=$ $\sqrt{2} I_{o} \sin \omega t$, with angular frequency $\omega$ and rms value $I_{0}$, the harmonic response coefficients $R_{n}$ are obtained by measuring the different frequency components $(1 \omega, 2 \omega, \ldots)$ with a lockin amplifier. The detected $n$th harmonic response at a set phase $\phi$ can be written as

$$
V_{n}(t)=\frac{\sqrt{2}}{T} \int_{t-T}^{t} \sin (n \omega s+\phi) V(s) d s .
$$

Focusing on first- and second-order responses, we define the output voltage of the lock-in amplifier for the first and second harmonic responses by using Eq. (B1) and Eq. (B2) as

$$
\begin{array}{ll}
V_{1}=I_{0} R_{1} & \text { for } \quad \phi=0^{\circ}, \\
V_{2}=I_{0}^{2} R_{2} / \sqrt{2} & \text { for } \quad \phi=-90^{\circ} .
\end{array}
$$

The SMR and SSE signals appear in the first and second harmonic responses in Eq. (B3), respectively.

In the linear response regime at currents $I \leqslant 2 \mathrm{~mA}$, the SMR scales linearly and the SSE scales quadratically with the applied current as shown in Figs. 6(a) and 6(b). However, at $I>2 \mathrm{~mA}$, the SMR (SSE) no longer depends linearly
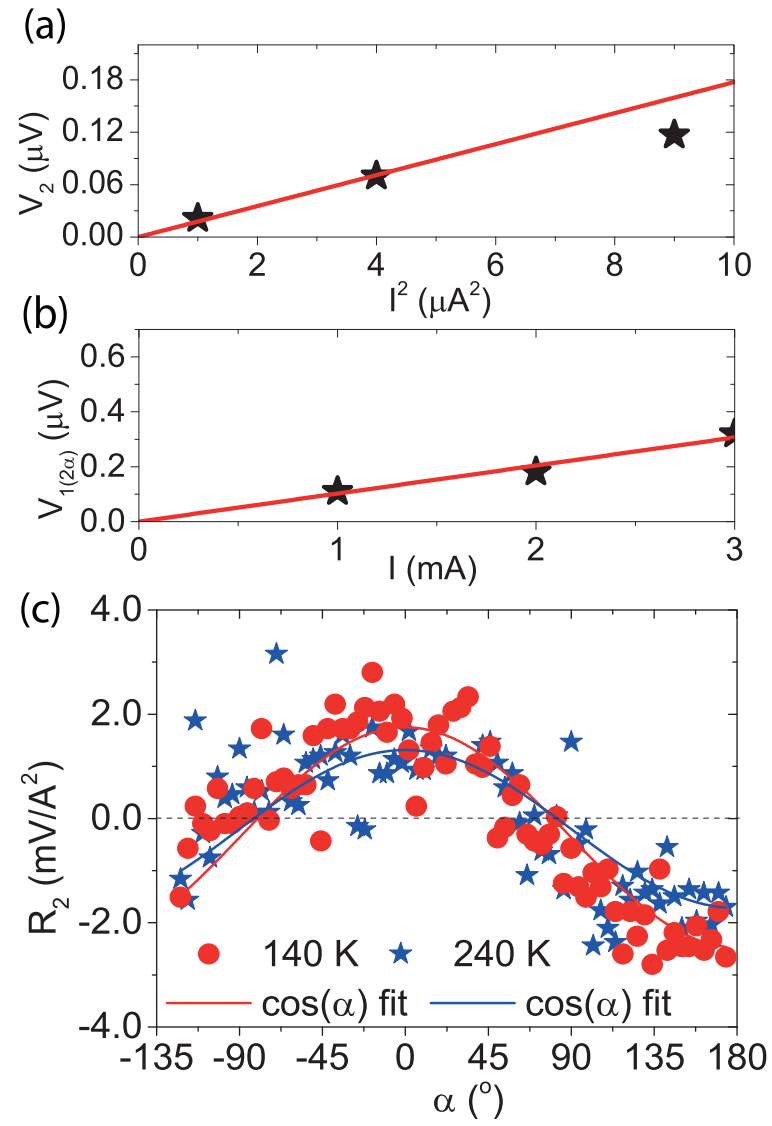

FIG. 6. (Color online) (a) Dependence of the second harmonic response $V_{2}$ on $I^{2}$, due to the SSE, generated by current-induced heating and (b) dependence of the first harmonic contribution $V_{1(2 \alpha)}$, due to SMR, by an ac current $I$ sent through the Pt Hall bar in a magnetic field of $4 \mathrm{~T}$. The SMR scales linearly and the SSE scales quadratically with $I$ in the linear regime $(I \leqslant 2 \mathrm{~mA})$. (c) Angular dependence of the second harmonic response, $R_{2}$, for $I=5 \mathrm{~mA}$, $H=4 \mathrm{~T}$ at $T=140$ and $240 \mathrm{~K}$. 

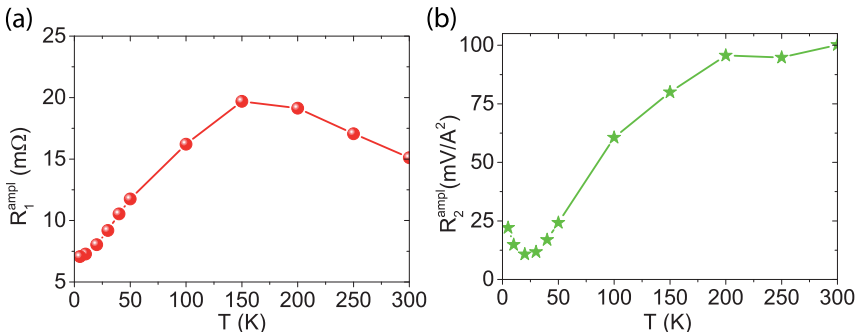

FIG. 7. (Color online) Temperature dependence of (a) the SMR and (b) the SSE in the Pt|YIG system for a 2.5-mA current through the Pt Hall-bar in a magnetic field of $0.1 \mathrm{~T}$.

(quadratically) on the current $I$. The results in the text for SMR are gathered in the linear regime. At $T>T_{s}$ the SSE signal decreases rapidly and we record the SSE also at the high current $I=5 \mathrm{~mA}$, as shown in Fig. 6(c).

\section{APPENDIX C: SMR AND THE SSE IN Pt|YIG VS Pt|CCO}

To compare the SMR and the SSE response in the Pt|CCO system to those in Pt|YIG, the angular dependence of the $\mathrm{Pt} \mid$ YIG system is also systematically studied at different temperatures. A $4 \mathrm{~nm}$ thick Pt Hall-bar was deposited on a $4 \times 4-\mathrm{mm}^{2}$ YIG film by dc sputtering [33]. The Pt Hall-bar has a length of $500 \mu \mathrm{m}$ and a width of $50 \mu \mathrm{m}$, with side contacts $10 \mu \mathrm{m}$ wide. A $200 \mathrm{~nm}$ thick, single-crystal YIG film is used and the film is grown by liquid phase epitaxy on a (111) $\mathrm{Gd}_{3} \mathrm{Ga}_{5} \mathrm{O}_{12}$ (GGG) substrate.

We observe the same sign of the SMR signals for Pt $\mid \mathrm{CCO}$ and Pt|YIG, as expected. A change in sign of the SSE has been observed in compensated ferrimagnets [39] but for both the Pt|YIG and the Pt|CCO systems the sign remains the normal for all temperatures (5-300 K).

The temperature dependence of the SMR and SSE is observed to be very different in the $\mathrm{Pt} \mid \mathrm{CCO}$ compared to the Pt|YIG system. In Pt|CCO, both SMR and SSE signals are enhanced at temperatures much lower than $T_{c}$, as shown in Figs. 2(e) and 4(c), with maximal values around $5 \mathrm{~K}$ in the spin lock-in phase. In contrast, Pt|YIG displays conventional behavior, with both the SMR and the SSE larger at room temperature than at low temperatures. Figure 7(a) shows the temperature dependence of Pt|YIG SMR at relatively high current levels, $I=2.5 \mathrm{~mA}$. The SMR signal increases slightly with decreasing temperature until $150 \mathrm{~K}$ and decreases again even more upon cooling [44]. The decrease in the SMR signal in Pt|YIG has been ascribed to a decrease in the Pt spin-Hall angle with decreasing temperature [45]. The SMR signal observed at $5 \mathrm{~K}$ is twice as small as that at room temperature. The SSE in Pt|YIG does not change much until $T=200 \mathrm{~K}$, while a further decrease in temperature suppresses the SSE as shown in Fig. 7(b). A small increase in the SSE is observed at $T<30 \mathrm{~K}$.

The ordinary Hall effect as observed in the first harmonic response of transverse resistance [as shown in Fig. 2(d)] is almost temperature independent in the $\mathrm{Pt} \mid \mathrm{CCO}$ system and scales linearly with the applied field as expected. The ordinary Hall effect is not observed in the Pt|YIG system because a much weaker field suffices to saturate the YIG magnetization $\left(H_{c}<1 \mathrm{mT}\right)$. Moreover, the SMR signal observed in Pt $\mid \mathrm{CCO}$ [shown in Fig. 2(e)] is smaller than that in the Pt|YIG system, resulting in a relatively larger contribution of the ordinary Hall effect. The SMR response observed at $5 \mathrm{~K}$ in $\mathrm{Pt} \mid \mathrm{CCO}$ is more than one order of magnitude smaller than the signal observed in the Pt|YIG system.
[1] C. W. Sandweg, Y. Kajiwara, A. V. Chumak, A. A. Serga, V. I. Vasyuchka, M. B. Jungfleisch, E. Saitoh, and B. Hillebrands, Phys. Rev. Lett. 106, 216601 (2011).

[2] V. Castel, N. Vlietstra, B. J. van Wees, and J. B. Youssef, Phys. Rev. B 86, 134419 (2012).

[3] C. Hahn, G. de Loubens, M. Viret, O. Klein, V. V. Naletov, and J. Ben Youssef, Phys. Rev. Lett. 111, 217204 (2013).

[4] K. Harii, T. An, Y. Kajiwara, K. Ando, H. Nakayama, T. Yoshino, and E. Saitoh, J. Appl. Phys. 109, 116105 (2011).

[5] K. Uchida, J. Xiao, H. Adachi, J. Ohe, S. Takahashi, J. Ieda, T. Ota, Y. Kajiwara, H. Umezawa, H. Kawai, G. E. W. Bauer, S. Maekawa, and E. Saitoh, Nature Mater. 9, 894 (2010).

[6] M. Schreier, A. Kamra, M. Weiler, J. Xiao, G. E. W. Bauer, R. Gross, and S. T. B. Goennenwein, Phys. Rev. B 88, 094410 (2013).

[7] A. Aqeel, I. J. Vera-Marun, B. J. van Wees, and T. T. M. Palstra, J. Appl. Phys. 116, 153705 (2014).

[8] S. M. Wu, J. E. Pearson, and A. Bhattacharya, Phys. Rev. Lett. 114, 186602 (2015).

[9] J. Flipse, F. K. Dejene, D. Wagenaar, G. E. W. Bauer, J. B. Youssef, and B. J. van Wees, Phys. Rev. Lett. 113, 027601 (2014).
[10] M. Althammer, S. Meyer, H. Nakayama, M. Schreier, S. Altmannshofer, M. Weiler, H. Huebl, S. Geprägs, M. Opel, R. Gross, D. Meier, C. Klewe, T. Kuschel, J.-M. Schmalhorst, G. Reiss, L. Shen, A. Gupta, Y.-T. Chen, G. E. W. Bauer, E. Saitoh, and S. T. B. Goennenwein, Phys. Rev. B 87, 224401 (2013).

[11] N. Vlietstra, J. Shan, V. Castel, B. J. van Wees, and J. Ben Youssef, Phys. Rev. B 87, 184421 (2013).

[12] H. Nakayama, M. Althammer, Y.-T. Chen, K. Uchida, Y. Kajiwara, D. Kikuchi, T. Ohtani, S. Geprägs, M. Opel, S. Takahashi, R. Gross, G. E. W. Bauer, S. T. B. Goennenwein, and E. Saitoh, Phys. Rev. Lett. 110, 206601 (2013).

[13] N. Vlietstra, J. Shan, V. Castel, J. Ben Youssef, G. E. W. Bauer, and B. J. van Wees, Appl. Phys. Lett. 103, 032401 (2013).

[14] C. Hahn, G. de Loubens, O. Klein, M. Viret, V. V. Naletov, and J. Ben Youssef, Phys. Rev. B 87, 174417 (2013).

[15] M. Isasa, A. Bedoya-Pinto, S. Vélez, F. Golmar, F. Sánchez, L. E. Hueso, J. Fontcuberta, and F. Casanova, Appl. Phys. Lett. 105, 142402 (2014).

[16] J. H. Han, C. Song, F. Li, Y. Y. Wang, G. Y. Wang, Q. H. Yang, and F. Pan, Phys. Rev. B 90, 144431 (2014).

[17] M. Fiebig, J. Phys. D: Appl. Phys. 38, R123 (2005).

[18] S.-W. Cheong and M. Mostovoy, Nature Mater. 6, 13 (2007).

[19] Y. Tokura and S. Seki, Adv. Mater. 22, 1554 (2010). 
[20] R. Ramesh and N. A. Spaldin, Nature Mater. 6, 21 (2007).

[21] T. Kimura and Y. Tokura, J. Phys.: Condens. Matter 20, 434204 (2008).

[22] Y. Yamasaki, S. Miyasaka, Y. Kaneko, J.-P. He, T. Arima, and Y. Tokura, Phys. Rev. Lett. 96, 207204 (2006).

[23] Y. J. Choi, J. Okamoto, D. J. Huang, K. S. Chao, H. J. Lin, C. T. Chen, M. van Veenendaal, T. A. Kaplan, and S.-W. Cheong, Phys. Rev. Lett. 102, 067601 (2009).

[24] K. Tomiyasu, J. Fukunaga, and H. Suzuki, Phys. Rev. B 70, 214434 (2004).

[25] S. Bordács, D. Varjas, I. Kézsmárki, G. Mihály, L. Baldassarre, A. Abouelsayed, C. A. Kuntscher, K. Ohgushi, and Y. Tokura, Phys. Rev. Lett. 103, 077205 (2009).

[26] S. Yang, H. X. Bao, D. Z. Xue, C. Zhou, J. H. Gao, Y. Wang, J. Q. Wang, X. P. Song, Z. B. Sun, X. B. Ren, and K. Otsuka, J. Phys. D: Appl. Phys. 45, 265001 (2012).

[27] G. Lawes, B. Melot, K. Page, C. Ederer, M. A. Hayward, T. Proffen, and R. Seshadri, Phys. Rev. B 74, 024413 (2006).

[28] K. Singh, A. Maignan, C. Simon, and C. Martin, Appl. Phys. Lett. 99, 172903 (2011).

[29] N. Mufti, A. A. Nugroho, G. R. Blake, and T. T. M. Palstra, J. Phys. Condens. Matter 22, 075902 (2010).

[30] K. R. Choi, S. J. Moon, T. Kouh, I. B. Shim, S. J. Kim, and C. S. Kim, IEEE Trans. Magn. 45, 2610 (2009).

[31] X. Liu, M. Kareev, Y. Cao, J. Liu, S. Middey, D. Meyers, J. W. Freeland, and J. Chakhalian, Appl. Phys. Lett. 105, 042401 (2014).

[32] V. Tsurkan, S. Zherlitsyn, S. Yasin, V. Felea, Y. Skourski, J. Deisenhofer, H.-A. K. von Nidda, J. Wosnitza, and A. Loidl, Phys. Rev. Lett. 110, 115502 (2013).

[33] N. Vlietstra, J. Shan, B. J. van Wees, M. Isasa, F. Casanova, and J. Ben Youssef, Phys. Rev. B 90, 174436 (2014).
[34] M. Schreier, N. Roschewsky, E. Dobler, S. Meyer, H. Huebl, R. Gross, and S. T. B. Goennenwein, Appl. Phys. Lett. 103, 242404 (2013).

[35] Y.-T. Chen, S. Takahashi, H. Nakayama, M. Althammer, S. T. B. Goennenwein, E. Saitoh, and G. E. W. Bauer, Phys. Rev. B 87, 144411 (2013).

[36] X. Jia, K. Liu, K. Xia, and G. E. W. Bauer, Europhys. Lett. 96, 17005 (2011).

[37] Y. Shiomi and E. Saitoh, Phys. Rev. Lett. 113, 266602 (2014).

[38] Y. Ohnuma, H. Adachi, E. Saitoh, and S. Maekawa, Phys. Rev. B 87, 014423 (2013).

[39] S. Geprägs, A. Kehlberger, T. Schulz, C. Mix, F. D. Coletta, S. Meyer, A. Kamra, M. Althammer, G. Jakob, H. Huebl, R. Gross, S. T. Goennenwein, and M. Kläui, arXiv:1405.4971[condmat.mes-hall].

[40] J. Barker, unpublished (2015).

[41] K. I. Uchida, T. Kikkawa, A. Miura, J. Shiomi, and E. Saitoh, Phys. Rev. X 4, 041023 (2014).

[42] D. Kamenskyi, H. Engelkamp, T. Fischer, M. Uhlarz, J. Wosnitza, B. P. Gorshunov, G. A. Komandin, A. S. Prokhorov, M. Dressel, A. A. Bush, V. I. Torgashev, and A. V. Pronin, Phys. Rev. B 87, 134423 (2013).

[43] V. I. Torgashev, A. S. Prokhorov, G. A. Komandin, E. S. Zhukova, V. B. Anzin, V. M. Talanov, L. M. Rabkin, A. A. Bush, M. Dressel, and B. P. Gorshunov, Phys. Solid State 54, 350 (2012).

[44] S. R. Marmion, M. Ali, M. McLaren, D. A. Williams, and B. J. Hickey, Phys. Rev. B 89, 220404 (2014).

[45] S. Meyer, M. Althammer, S. Geprägs, M. Opel, R. Gross, and S. T. B. Goennenwein, Appl. Phys. Lett. 104, 242411 (2014). 